\title{
On Complexity of Ehrenfeucht-Fraïssé Games
}

\author{
Bakhadyr Khoussainov ${ }^{1}$ and Jiamou Liu ${ }^{2}$ \\ 1 Department of Computer Science \\ University of Auckland \\ New Zealand \\ 2 Department of Computer Science \\ University of Auckland \\ New Zealand
}

\begin{abstract}
In this paper we initiate the study of Ehrenfeucht-Fraïssé games for some standard finite structures. Examples of such standard structures are equivalence relations, trees, unary relation structures, Boolean algebras, and some of their natural expansions. The paper concerns the following question that we call Ehrenfeucht-Fraïssé problem. Given $n \in \omega$ as a parameter, two relational structures $\mathcal{A}$ and $\mathcal{B}$ from one of the classes of structures mentioned above, how efficient is it to decide if Duplicator wins the $n$-round EF game $G_{n}(\mathcal{A}, \mathcal{B})$ ? We provide algorithms for solving the Ehrenfeucht-Fraïssé problem for the mentioned classes of structures. The running times of all the algorithms are bounded by constants. We obtain the values of these constants as functions of $n$.
\end{abstract}

\section{Introduction}

Ehrenfeucht-Fraïssé (EF) games constitute an important tool in both finite and infinite model theory. For example, in infinite model theory these games can be used to prove Scott Isomorphism Theorem showing that all countable structures are described (up to isomorphism) in $L_{\omega_{1}, \omega}$-logic. In finite model theory these games and their different versions are used for establishing expressibility results in the first order logic and its extensions. These results can be found in standard books in finite and infinite model theory (e.g. [6], [11]) or relatively recent papers (e.g. [2], [12]). In this paper all EF games are considered on finite structures.

Despite significant use of EF games in finite and infinite model theory there has not been, with some exceptions, much work in addressing efficiency of these games. M. Grohe studied EF games with fixed number of pebbles and showed that the problem of deciding the winner is complete for PTIME [5]. E. Pezzoili showed that deciding the winner of EF games is PSPACE-complete [14]. In [9] P. Kolaitis and J. Panttaja prove that the following problem is EXPTIME-complete: given a natural number $k$ and structures $\mathcal{A}$ and $\mathcal{B}$, does Duplicator win the $k$ pebble existential EF game on $\mathcal{A}$ and $\mathcal{B}$ ? In [1] sufficient conditions are provided for Duplicator to win EF games. These conditions are then used to prove some inexpressibility results, e.g reachability in undirected graphs is not in monadic NP. These results suggest that developing tools and algorithms for finding winners of EF are of interest. We also point out that there has recently been an interest in EF games to collapse results in database theory [16]. In addition, we think that algorithms that solve EF games can be used in data matching and data transformation problems in databases.

In this paper we initiate the study of EF games for some standard finite structures. Examples of such standard structures are equivalence relations, trees, unary relation structures, 
Boolean algebras, and some of their natural expansions. The paper concerns the following question that we call the Ehrenfeucht-Fraïssé problem. Given $n \in \omega$ as a parameter, two relational structures $\mathcal{A}$ and $\mathcal{B}$, how efficient is it to decide if Duplicator wins the $n$-round EF game $G_{n}(\mathcal{A}, \mathcal{B})$ ? We provide algorithms for solving the Ehrenfeucht-Fraïssé problem for the structures mentioned above. The running times of all the algorithms are bounded by constants. We obtain the values of these constants as functions of $n$.

By a structure we always mean a finite relational structure over a language without functional symbols. Let $\mathcal{A}$ and $\mathcal{B}$ be structures and $n \in \omega$. EF game, denoted by $G_{n}(\mathcal{A}, \mathcal{B})$, on these two structures is played as follows. There are two players, Duplicator and Spoiler, both provided with $\mathcal{A}$ and $\mathcal{B}$. The game consists of $n$ rounds. Informally, Duplicator's goal is to show that these two structures are similar, while Spoiler needs to show the opposite. At round $i$, Spoiler selects structure $\mathcal{A}$ or $\mathcal{B}$, and then takes an element from the selected structure. Duplicator responds by selecting element from the other structure. Say, the players have produced the following play consisting of pairs of elements $\left(a_{1}, b_{1}\right), \ldots,\left(a_{n}, b_{n}\right)$, where $a_{i} \in A$ and $b_{i} \in B$ for $i=1, \ldots, n$. Note that if Spoiler selected $a_{i}$ (or $b_{i}$ ) then Duplicator selected $b_{i}$ (or $a_{i}$, respectively). Duplicator wins the play if the mapping $a_{i} \rightarrow b_{i}, i=1, \ldots, n$, extended by mapping the values of constant symbols $c^{\mathcal{A}}$ to $c^{\mathcal{B}}$, is a partial isomorphism between $\mathcal{A}$ and $\mathcal{B}$. It is clear that if $\mathcal{A}$ and $\mathcal{B}$ are isomorphic then Duplicator wins the game $G_{n}(\mathcal{A}, \mathcal{B})$ no matter what $n$ is. The opposite is not always true. However, for large $n$ if Duplicator wins the game $G_{n}(\mathcal{A}, \mathcal{B})$ then $\mathcal{A}$ and $\mathcal{B}$ are isomorphic. Thus, solving the EF problem can be thought as an approximation to the isomorphism problem.

One can do the following rough estimates for finding the winner of the game $\mathcal{G}_{n}(\mathcal{A}, \mathcal{B})$. There are finitely many, up to logical equivalence, formulas $\phi_{1}, \ldots, \phi_{k}$ of quantifier rank $n$ (see for example [11]). It is well known that Duplicator wins $G_{n}(\mathcal{A}, \mathcal{B})$ if and only if for all $\phi_{i}$ ( with $i=1, \ldots, k$ ) the structure $\mathcal{A}$ satisfies $\phi_{i}$ if and only if $\mathcal{B}$ satisfies $\phi_{i}$ [11]. Thus, the question if Duplicator wins $G_{n}(\mathcal{A}, \mathcal{B})$ can be solved in polynomial time. However, there are two important issues here. The first issue concerns the number $k$ that depends on $n ; k$ is approximately bounded by the $n$-repeated exponentiations of 2 . The second issue concerns the degree of the polynomial for the running time that is bounded by $n$. Thus, the questions arise as to for which standard structures the value of $k$ is feasible as a function of $n$, and whether the degree of the polynomial for the running time can be pushed down. As an example consider the class of linear orders. It is well-known that Duplicator wins $G_{n}(\mathcal{A}, \mathcal{B})$, where $\mathcal{A}$ and $\mathcal{B}$ are linear orders, if and only if either $|A|=|B|$ or both $|A|>2^{n}$ and $|B|>2^{n}$ (e.g. [11]). In this example, the number $k$, roughly, equals to $2^{n}$. The degree of polynomial for the running time is 0 . Thus, when $n$ is fixed the winner of the game can be found in constant time, and the constant that bounds the time is $2^{n}$.

A brief overview of this paper is as follows. The next section gives an elementary solution to EF games in the case when the language contains unary predicates only. The third, fourth and fifth sections are quite technical and devoted to solving EF games for equivalence structures and some of their extensions. Equivalence structures are natural models of university or large company databases. For example, in a university database there could be the SameFaculty and the SameDepartment relations. The first relation stores all tuples $(x, y)$ such that 
$x$ and $y$ belong to the same faculty; similarly, the second relation stores all tuples $(u, v)$ such that $u$ and $v$ are in the same department. These relations are equivalence relations. Moreover, the set-theoretic connection between these relations is that the relation SameDepartment is a subset of the SameFaculty relation. We call such structures embedded equivalence relation structures. Section 6 reduces the question of deciding EF games for trees of a given height to solving the EP games for embedded equivalence structures introduced in the previous sections. Finally, the main structures in the last section are Boolean algebras with distinguished ideals.

Each of these sections provides an algorithm that decides EF games $G_{n}(\mathcal{A}, \mathcal{B})$, where $\mathcal{A}$ and $\mathcal{B}$ are structures considered in the section. These algorithms run in constant times with $n$ being a parameter. We also bound the value of the constants as a function of $n$. Clearly, the constants obtained depend on the representations of the structures. In each case, it will be clear from the content how we represent our structures. As an example we state two results of Sections 4 and 5. Section 4 is devoted to structures of the type $\left(A ; E, P_{1}, \ldots, P_{s}\right)$, where $E$ is an equivalence relation on $A$ and $P_{1}, \ldots, P_{s}$ are unary predicates. We call these structures equivalence structures with $s$ colors. The main result of Section 4 is the following:

Theorem 5 Fix $n \in \omega$. There exists an algorithm that runs in constant time and decides whether Duplicator wins the game $G_{n}(\mathcal{A}, \mathcal{B})$ on equivalence structures with s colors. The constant that bounds the running time is $n^{2^{s}+1}$.

Section 5 is devoted to the structures of type $\left(A ; E_{1}, \ldots, E_{h}\right)$, where each $E_{i}$ is an equivalence relation on $A$ and $E_{1} \subseteq E_{2} \subseteq \ldots \subseteq E_{h}$. These structures are called embedded equivalence structures of height $h$. The main result of Section 5 is:

Theorem 7 Fix $n \in \omega$. There exists an algorithm that runs in constant time and decides whether Duplicator wins the game $G_{n}(\mathcal{A}, \mathcal{B})$ on embedded equivalence structures of height $h$. The constant that bounds the running time is $(n+1)^{\cdots^{(n+1)^{n}}}$ where the tower has height $h$.

\section{Simple Example: Structures With Unary Predicates}

This is an elementary section that gives a full solution for EF games in the case when the language contains unary predicates only. Here is the main result of this section.

Theorem 1. Fix the language $L=\left(P_{1}, \ldots, P_{s}\right)$, where each $P_{i}$ is a unary predicate symbol. Let $n \in \omega$. There exists an algorithm that runs in constant time and decides whether Duplicator wins the game $G_{n}(\mathcal{A}, \mathcal{B})$ on structures $\mathcal{A}$ and $\mathcal{B}$ of the language. The constant that bounds the running time is $2^{s} \cdot n$.

Proof. Let $\mathcal{A}=\left(A ; P_{1}, P_{2}, \ldots, P_{s}\right)$ and $\mathcal{B}=\left(B ; P_{1}, P_{2}, \ldots, P_{s}\right)$ be structures of the language given. For structure $\mathcal{A}=\left(A ; P_{1}, P_{2}, \ldots, P_{s}\right)$, we set $P_{s+1}=\bigcap_{i} \neg P_{i}$.

Lemma 1. Suppose $P_{1}, P_{2}, \ldots, P_{s}$ are pairwise disjoint. Then Duplicator wins $G_{n}(\mathcal{A}, \mathcal{B})$ if and only if for all $1 \leq i \leq s+1$ if $\left|P_{i}^{A}\right|<n$ or $\left|P_{i}^{B}\right|<n$ then $\left|P_{i}^{A}\right|=\left|P_{i}^{B}\right|$. In particular, when Duplicator wins it is the case that for all $1 \leq i \leq s+1,\left|P_{i}^{A}\right| \geq n$ if and only if $\left|P_{i}^{B}\right| \geq n$. 
To prove the lemma suppose that there is $1 \leq i \leq k+1$ such that $\left|P_{i}^{A}\right|<n$ but $\left|P_{i}^{A}\right| \neq\left|P_{i}^{B}\right|$. Assume $\left|P_{i}^{B}\right|<\left|P_{i}^{A}\right|$. Then Spoiler selects $\left|P_{i}^{A}\right|$ elements from $P_{i}^{A}$. This strategy is clearly a winning strategy for Spoiler. For the other direction, assume that hypothesis of the lemma holds. Duplicator has a winning strategy as follows. At round $k$, assume that the players have produced the $k$-round play $\left(a_{1}, b_{1}\right), \ldots,\left(a_{k}, b_{k}\right)$ such that for each $1 \leq i \leq k, a_{i} \in A, b_{i} \in B$. If Spoiler selects $a_{k+1} \in A$, then Duplicator responds by selecting $b_{k+1} \in B$ as follows: If $a_{k+1}=a_{i}$ for some $i$ then $b_{k+1}=b_{i}$. Otherwise if $a_{k+1} \in P_{j}^{A}$ for some $1 \leq j \leq k$, then $b_{k+1} \in P_{j}^{B}$ so that $b_{k+1} \notin\left\{b_{1}, \ldots, b_{k}\right\}$. The case when Spoiler selects an element from $B$ is treated similarly. The strategy is clearly winning.

Now assume that for a structure $\mathcal{A}$, the unary predicates $P_{1}, P_{2}, \ldots, P_{s}$ are not necessarily pairwise disjoint. For each element $x \in A$, define the characteristic of $x, \operatorname{ch}(x)$, as a binary sequence $\left(t_{1}, t_{2}, \ldots, t_{s}\right)$ such that for each $1 \leq i \leq s, t_{i} \in\{0,1\}$ if $x \in P_{i}$ and $t_{i}=0$ otherwise. There are $2^{s}$ pairwise distinct characteristics, and we order them in lexicographic order: $c h_{1}, \ldots, c h_{2^{s}}$. Construct the structure $\mathcal{A}^{\prime}=\left(A ; Q_{1}, \ldots, Q_{2^{s}}\right)$ such that for all $1 \leq i \leq 2^{s}$, $Q_{i}=\left\{x \in A \mid \operatorname{ch}(x)=c h_{i}\right\}$. The following is now an easy lemma.

Lemma 2. Duplicator wins $G_{n}(\mathcal{A}, \mathcal{B})$ if and only if Duplicator wins $G_{n}\left(\mathcal{A}^{\prime}, \mathcal{B}^{\prime}\right)$.

We now represent $\mathcal{A}$ and $\mathcal{B}$ by $2^{s}$ lists, and the $i^{\text {th }}$ list lists all elements with characteristic $c h_{i}$. To solve the game $G_{n}\left(\mathcal{A}^{\prime}, \mathcal{B}^{\prime}\right)$, the algorithm checks the conditions in Lemma 1 by reading the lists. The process takes time bounded by $2^{s} \cdot n$ as required.

\section{Equivalence Structures}

An equivalence structure is a structure $\mathcal{A}$ of the type $(A ; E)$ where $E$ is an equivalence relation on $A$. We list all the equivalence classes of $\mathcal{A}$ as $A_{1}, \ldots, A_{k}$ such that $\left|A_{i}\right| \leq\left|A_{i+1}\right|$ for all $1 \leq i<k$. Let $q_{\mathcal{A}}$ be the number of equivalent classes in $\mathcal{A}$; for each $t<n$, let $q_{t}^{A}$ be the number of equivalence classes in $\mathcal{A}$ with size $t$. Finally, let $q_{\geq r}^{\mathcal{A}}$ be the number of equivalence classes in $\mathcal{A}$ of size at least $r$. For an equivalence structure $\mathcal{B}$ we have similar notations as $B_{1}$, $B_{2}, \ldots$ to denote its equivalence classes, and the associated numbers $q_{\mathcal{B}}, q_{t}^{\mathcal{B}}$, and $q_{\geq r}^{\mathcal{B}}$.

Lemma 3. If Duplicator wins the game $G_{n}(\mathcal{A}, \mathcal{B})$ on equivalence structures $\mathcal{A}$ and $\mathcal{B}$, then the following must be true:

1. If $q_{\mathcal{A}}<n$ or $q_{\mathcal{B}}<n$ then $q_{\mathcal{A}}=q_{\mathcal{B}}$; and

2. $q_{\mathcal{A}} \geq n$ if and only if $q_{\mathcal{B}} \geq n$.

In our analysis below, by the above lemma, we always assume that $q_{\mathcal{A}}=q_{\mathcal{B}}$ or $q_{\mathcal{A}} \geq n$ if and only if $q_{\mathcal{B}} \geq n$. We need the following notation for the next lemma and definition. For $t \leq n$, let $q^{t}=\min \left\{q_{\geq t}^{\mathcal{A}}, q_{\geq t}^{\mathcal{B}}\right\}$. Let $\mathcal{A}_{t}$ and $\mathcal{B}_{t}$ be equivalence structures obtained by taking out exactly $q^{t}$ equivalence classes of size $\geq t$ from $\mathcal{A}$ and $\mathcal{B}$ respectively. We also set $n-q^{t}$ to be 0 in case $q^{t} \geq n$; and otherwise $n-q^{t}$ has its natural meaning.

Lemma 4. 1. Assume that there is a $t<n$ such that $q_{t}^{\mathcal{A}} \neq q_{t}^{\mathcal{B}}$ and $n-q^{t}>t$. Then Spoiler wins the game $G_{n}(\mathcal{A}, \mathcal{B})$. 
2. Assume that there is a $t \leq n$ such that $n-q^{t}>0$ and one of the structures $\mathcal{A}_{t}$ or $\mathcal{B}_{t}$ has an equivalence class of size $\geq n-q^{t}$ and the other structure does not. Then Spoiler wins the game $G_{n}(\mathcal{A}, \mathcal{B})$

Proof. We prove the first part of the lemma. The second part is proved similarly. Assume, without loss of generality, that $q_{t}^{\mathcal{A}}>q_{t}^{\mathcal{B}}$ and $n-q_{t}^{\mathcal{B}}>t$. Spoiler's strategy is the following. First, select elements $a_{1}, \ldots, a_{q_{t}^{\mathcal{B}}}$ from distinct equivalence classes of size $t$ in $\mathcal{A}$. Next, select $t$ distinct elements in the equivalence class of size $t$ in $\mathcal{A}$. This leads Spoiler to win.

Definition 1. 1. We say that $G_{n}(\mathcal{A}, B)$ has small disparity if there is a $t<n$ such that either $q_{t}^{\mathcal{A}} \neq q_{t}^{\mathcal{B}}$ and $n-q^{t}>t$.

2. We say that $G_{n}(\mathcal{A}, B)$ has large disparity if there exists a $t \leq n$ such that $n-q^{t}>0$ and one of the structures $\mathcal{A}_{t}$ or $\mathcal{B}_{t}$ has an equivalence class of size $\geq n-q^{t}$ and the other structure does not.

Lemma 5. Duplicator wins the game $G_{n}(\mathcal{A}, \mathcal{B})$ if and only if the game $G_{n}(\mathcal{A}, \mathcal{B})$ has neither small nor large disparity.

Proof. The previous lemma proves one direction. For the other, we assume that neither small nor large disparity occurs in the game. We describe a winning strategy for Duplicator.

Let us a assume that the players have produced a $k$-round play $\left(a_{1}, b_{1}\right),\left(a_{2}, b_{2}\right), \ldots,\left(a_{k}, b_{k}\right)$. In case $k=0$, we are at the start of the game $G_{n}(\mathcal{A}, \mathcal{B})$. Our inductive assumptions on this $k$-round play are the following:

1. $E\left(a_{i}, a_{j}\right)$ is true in $\mathcal{A}$ if and only if $E\left(b_{i}, b_{j}\right)$ is true in $\mathcal{B}$, and the map $a_{i} \rightarrow b_{i}$ is one-to-one.

2. For all $a_{i},\left|\left[a_{i}\right]\right| \geq n-i$ if and only if $\left|\left[b_{i}\right]\right| \geq n-i$, where $[x]$ denotes the equivalence class of $x$.

3. For $a_{i}$ if $\left|\left[a_{i}\right]\right|<n-i$ then $\left|\left[a_{i}\right]\right|=\left|\left[b_{i}\right]\right|$.

4. Let $\mathcal{A}^{\prime}$ and $\mathcal{B}^{\prime}$ be the equivalence structures obtained by removing the equivalence classes $\left[a_{1}\right], \ldots,\left[a_{k}\right]$ from $\mathcal{A}$ and the equivalence classes $\left[b_{1}\right], \ldots,\left[b_{k}\right]$ from $\mathcal{B}$, respectively. We assume that $\mathcal{A}^{\prime}$ and $\mathcal{B}^{\prime}$ satisfy the following conditions:

(a) In game $G_{n-k}\left(\mathcal{A}^{\prime}, \mathcal{B}^{\prime}\right)$ no small disparity occurs.

(b) In game $G_{n-k}\left(\mathcal{A}^{\prime}, \mathcal{B}^{\prime}\right)$ no large disparity occurs.

Assume that Spoiler selects $a_{k+1} \in A$. Duplicator responds by choosing $b_{k+1}$ as follows. If $a_{k+1}=a_{i}$ then $b_{k+1}=b_{i}$. Otherwise, if $E\left(a_{i}, a_{k+1}\right)$ is true in $\mathcal{A}$ then Duplicator chooses a new $b_{k+1}$ such that $E\left(b_{i}, b_{k+1}\right)$ is true in $\mathcal{B}$. Assume $a_{k+1}$ is not equivalent to any of the elements $a_{1}, \ldots, a_{k}$. If $\left|\left[a_{k+1}\right]\right| \geq n-k$ then Duplicator chooses $b_{k+1}$ such that $b_{k+1}$ is not equivalent to any of the elements $b_{1}, \ldots, b_{k}$ and $\left|\left[b_{k+1}\right]\right| \geq n-k$. Duplicator can select such an element as otherwise large disparity would occur in the game. If $\left|\left[a_{k+1}\right]\right|<n-k$ then Duplicator chooses $b_{k+1}$ such that $\left|\left[b_{k+1}\right]\right|=\left|\left[a_{k+1}\right]\right|$ and $b_{k+1}$ is not equivalent to any of the elements $b_{1}, \ldots, b_{k}$. The case when Spoiler selects an element from $B$ is treated similarly.

Now we show that the $(k+1)$-round play $\left(a_{1}, b_{1}\right),\left(a_{2}, b_{2}\right), \ldots,\left(a_{k}, b_{k}\right),\left(a_{k+1}, b_{k+1}\right)$ satisfies the inductive assumptions. The inductive assumptions (1), (2), and (3) can easily be checked 
to be preserved. To show that the assumption (4) is preserved, consider the equivalence structures $\mathcal{A}^{\prime \prime}$ and $\mathcal{B}^{\prime \prime}$ obtained by removing the equivalence classes $\left[a_{1}\right], \ldots,\left[a_{k}\right],\left[a_{k+1}\right]$ from $\mathcal{A}$ and the equivalence classes $\left[b_{1}\right], \ldots,\left[b_{k}\right],\left[b_{k+1}\right]$ from $\mathcal{B}$, respectively. In game $G_{n-k-1}\left(\mathcal{A}^{\prime \prime}, \mathcal{B}^{\prime \prime}\right)$ small disparity does not occur as otherwise the game $G_{n-k}\left(\mathcal{A}^{\prime}, \mathcal{B}^{\prime}\right)$ would have small disparity. Thus, assumption ( $4 a$ ) is also preserved. Similarly, if $G_{n-k-1}\left(\mathcal{A}^{\prime \prime}, \mathcal{B}^{\prime \prime}\right)$ had large disparity then the game $G_{n-k}\left(\mathcal{A}^{\prime}, \mathcal{B}^{\prime}\right)$ would also have large disparity contradicting the inductive assumption. Hence, the strategy described must be a winning strategy due to the fact that Duplicator preserves the inductive assumption (1) at each round.

For the next theorem, we represent each equivalence structure $\mathcal{A}$ and $\mathcal{B}$ in two lists. For example, the first list for the structure $\mathcal{A}$ lists all equivalence classes of $\mathcal{A}$ in increasing order; the second list is $q^{\mathcal{A}}, q_{1}^{\mathcal{A}}, q_{\geq 1}^{\mathcal{A}}, q_{2}^{\mathcal{A}}, q_{\geq 2}^{\mathcal{A}}, \ldots$ The lemmas above give us the following:

Theorem 2. Fix $n \in \omega$. There exists an algorithm that runs in constant time and decides whether Duplicator wins the game $G_{n}(\mathcal{A}, \mathcal{B})$ on equivalence structures $\mathcal{A}=(A ; E)$ and $\mathcal{B}=$ $(B ; E)$. The constant that bounds the running time is $n$.

We can extend the above theorem by defining the following structures:

Definition 2. A homogeneous equivalence structure is $\left(A ; E, P_{1}, \ldots, P_{s}\right)$ such that

- $(A ; E)$ is an equivalence structure; and

- Each $P_{i}$ is a homogeneous unary relation on A meaning that for all $x, y \in A$ if $E(x, y)$ then $x \in P_{i}$ if and only if $y \in P_{i}$.

For a homogeneous equivalence structure $\mathcal{A}$, define the characteristic $\operatorname{ch}(x)$ of an element $x \in A$ as in Section 2. Represent $\mathcal{A}$ as a disjoint union of equivalence structures $\mathcal{A}_{1}, \ldots, \mathcal{A}_{2^{s}}$, where $A_{\epsilon}$ consists of elements with characteristic $\epsilon$. The above theorem is thus extended to:

Theorem 3. There exists an algorithm that runs in constant time and decides whether Duplicator wins the game $G_{n}(\mathcal{A}, \mathcal{B})$ on homogeneous equivalence structures $\mathcal{A}$ and $\mathcal{B}$. The constant that bounds the running time is $2^{s} \cdot n$.

\section{Equivalence Structures With Colors}

In this section structures $\mathcal{A}$ are of the form $\left(A ; E, P_{1}, \ldots, P_{s}\right)$, where $E$ is an equivalence relation on $A$ and $P_{1}, \ldots, P_{s}$ are unary predicates on $A$. We call these equivalence structures with $s$ colors. We start with the case when $s=1$. The case for $s>2$ will be explained later.

Let $\mathcal{A}=(A ; E, P)$ be a equivalence structure with one color. Say $x \in A$ is colored if $P(x)$ is true; otherwise $x$ is non-colored. An equivalence class $X$ has type $\operatorname{tp}(X)=(i, j)$, if the number of colored elements of $X$ is $i$, non-colored elements is $j$; thus, $i+j=|X|$.

Definition 3. Given two types $(i, j)$ and $\left(i^{\prime}, j^{\prime}\right)$ respectively. We say that $(i, j)$ is colored $n$ equivalent to $\left(i^{\prime}, j^{\prime}\right)$, denoted by $(i, j) \equiv_{n}^{C}\left(i^{\prime}, j^{\prime}\right)$, if the following holds.

1. If $i<n$ then $i^{\prime}=i$; otherwise $i^{\prime} \geq n$. 
2. If $j<n-1$ then $j^{\prime}=j$; otherwise $j^{\prime} \geq n-1$.

We say that $(i, j)$ is non-colored $n$-equivalent to $\left(i^{\prime}, j^{\prime}\right)$, denoted by $(i, j) \equiv_{n}^{N}\left(i^{\prime}, j^{\prime}\right)$, if the following holds.

1. If $j<n$ then $j^{\prime}=j$; otherwise $j^{\prime} \geq n$.

2. If $i<n-1$ then $i^{\prime}=i$; otherwise $i^{\prime} \geq n-1$.

For $X \subseteq A$, we use $(X ; E \uparrow X, P \uparrow X)$ to denote the equivalence structure obtained by restricting $E$ and $P$ on $X$. Note that given two equivalence classes $X$ and $Y$ of types $(i, j)$ and $\left(i^{\prime}, j^{\prime}\right)$ respectively, if $(i, j)$ is colored (non-colored) $n$-equivalent to $\left(i^{\prime}, j^{\prime}\right)$, then Duplicator wins the $n$-round game played on structures $(X ; E \uparrow X, P \uparrow X)$ and $(Y, E \uparrow Y, P \uparrow Y)$, given the fact that Spoiler chooses a colored (non-colored) element in the first round.

Lemma 6. If either $\left(i^{\prime}, j^{\prime}\right) \equiv_{n}^{C}(i, j)$ or $\left(i^{\prime}, j^{\prime}\right) \equiv_{n}^{N}(i, j)$, then $\left(i^{\prime}, j^{\prime}\right) \equiv_{n-1}^{C}(i, j)$ and $\left(i^{\prime}, j^{\prime}\right) \equiv_{n-1}^{N}$ $(i, j)$.

For an equivalence structure $\mathcal{A}=(A ; E, P)$, we need the following notations:

- For type $(i, j)$ and $k \geq 1$, Set $C_{(i, j), k}^{\mathcal{A}}$ be the set $\{X \mid X$ is an equivalence class of $\mathcal{A}$ and $\left.\operatorname{tp}(X) \equiv_{k}^{C}(i, j)\right\}$. Set $N_{(i, j), k}^{\mathcal{A}}$ be the set $\left\{X \mid X\right.$ is an equivalence class of $\mathcal{A}$ and $\operatorname{tp}(X) \equiv_{k}^{N}$ $(i, j)\}$

- For type $(i, j)$ and $k \geq 1$, set $q_{(i, j), k}^{\mathcal{A}, C}=\left|C_{(i, j), k}^{\mathcal{A}}\right|$, and set $q_{(i, j), k}^{\mathcal{A}, N}=\left|N_{(i, j), k}^{\mathcal{A}}\right|$.

- For $\mathcal{A}$ and $\mathcal{B}$, set $q_{(i, j), k}^{C}=\min \left\{q_{(i, j), k}^{\mathcal{A}, C}, q_{(i, j), k}^{\mathcal{B}, C}\right\}$ and $q_{(i, j), k}^{N}=\min \left\{q_{(i, j), k}^{\mathcal{A}, N}, q_{(i, j), k}^{\mathcal{B}, N}\right\}$

- Set $\mathcal{A}^{C}((i, j), k)$ be the structure obtained from $\mathcal{A}$ by removing $q_{(i, j), k}^{C}$ equivalence classes in $C_{(i, j), k}^{\mathcal{A}}$.

- Set $\mathcal{A}^{N}((i, j), k)$ be the structure obtained from $\mathcal{A}$ by removing $q_{(i, j), k}^{N}$ equivalence classes in $N_{(i, j), k}^{\mathcal{A}}$.

Observe the following. If Spoiler selects a colored element from an equivalence class $X$ in $\mathcal{A}$, and Duplicator responds by selecting a colored element from an equivalence class $Y$ such that $t p(Y) \equiv_{n}^{C} t p(X)$, there is no point for Spoiler to play inside $X$ because this will guarantee a win for Duplicator. Conversely, suppose Spoiler selects a colored element from an equivalence class $X$ in $\mathcal{A}$, and there is no equivalence class in $\mathcal{B}$ whose type is colored $n$-equivalent to $t p(X)$. Then Spoiler has a winning strategy by playing inside $X$ and $Y$.

Definition 4. Consider the game $G_{n}(\mathcal{A}, \mathcal{B})$ played on equivalence structures with one color. We say that a colored disparity occurs if there exists a type $(i, j)$ and $n>k \geq 0$ such that the following holds:

1. $k=q_{(i, j), n-k}^{C}$.

2. In one of $\mathcal{A}^{C}((i, j), n-k)$ and $\mathcal{B}^{C}((i, j), n-k)$, there is an equivalence class whose type is colored $(n-k)$-equivalent to $(i, j)$, and no such equivalence class exists in the other structure.

We say that a non-colored disparity occurs if there exists a type $(i, j)$ and $n>k \geq 0$ such that the following holds: 
1. $k=q_{(i, j), n-k}^{N}$.

2. In one of $\mathcal{A}^{N}((i, j), n-k)$ and $\mathcal{B}^{N}((i, j), n-k)$, there is an equivalence class whose type is non-colored $(n-k)$-equivalent to $(i, j)$, and no such equivalence class exists in the other structure.

Lemma 7. Suppose $\mathcal{A}$ and $\mathcal{B}$ are two equivalence structures with one color. Duplicator wins the game $G_{n}(\mathcal{A}, \mathcal{B})$ if and only if neither colored disparity nor non-colored disparity occurs in the game.

Proof. If either colored or non-colored disparity occurs in the game, then it is not too hard to see that Spoiler wins the game. Suppose that neither colored disparity nor non-colored disparity occurs in the game $G_{n}(\mathcal{A}, \mathcal{B})$, we describe a strategy for Duplicator. Let us assume that the players have produced a $k$-round play $\left(a_{1}, b_{1}\right),\left(a_{2}, b_{2}\right), \ldots,\left(a_{k}, b_{k}\right)$. Let $\left(i_{l}, j_{l}\right)$ and $\left(i_{l}^{\prime}, j_{l}^{\prime}\right)$ be the types of $a_{l}$ and $b_{l}$,respectively with $1 \leq l \leq k$. Our inductive assumptions on this $k$ round play are the following:

1. For any $1 \leq l \leq k, a_{l}$ is a colored element if and only if $b_{l}$ is a colored element.

2. For any $1 \leq l, m \leq k, E\left(a_{l}, a_{m}\right)$ if and only if $E\left(b_{l}, b_{m}\right)$.

3. For any $1 \leq l \leq k,\left(i_{l}, j_{l}\right) \equiv_{n-l}^{C}\left(i_{l}^{\prime}, j_{l}^{\prime}\right)$ and $\left(i_{l}, j_{l}\right) \equiv_{n-l}^{N}\left(i_{l}^{\prime}, j_{l}^{\prime}\right)$.

4. Let $\mathcal{A}^{\prime}$ and $\mathcal{B}^{\prime}$ be the equivalence structures obtained by removing equivalence classes $\left[a_{1}\right], \ldots,\left[a_{k}\right]$ from $\mathcal{A}$ and $\left[b_{1}\right], \ldots,\left[b_{k}\right]$ from $\mathcal{B}$, respectively. We assume in game $G_{n-k}$ neither colored disparity nor non-colored disparity occurs.

Assume that Spoiler selects an element $a_{k+1} \in A$. Duplicator responds to this move by choosing $b_{k+1}$ as follows. If $a_{k+1}=a_{l}$ then $b_{k+1}=b_{l}$. Otherwise, if $E\left(a_{k+1}, a_{l}\right)$ is true in $\mathcal{A}$, then Duplicator chooses a new $b_{k+1}$ such that $E\left(b_{k+1}, b_{l}\right)$ and $a_{k+1}$ is a colored element if and only if $b_{k+1}$ is a colored element. By (3) of the inductive assumption, Duplicator can always select such an element $b_{k+1}$.

Assume $a_{k+1}$ is not equivalent to any of the element $a_{1}, \ldots, a_{k}$. Let $X$ be the equivalence class of $a_{k+1}$ in $\mathcal{A}$. If $a_{k+1}$ is a colored element, then Duplicator chooses a colored element $b_{k+1}$ from an equivalence class $Y$ of $\mathcal{B}$ such that $t p(X) \equiv_{n-k}^{C} t p(Y)$. If $a_{k+1}$ is a non-colored element, then Duplicator chooses a non-colored $b_{k+1}$ from an equivalence class $Y$ of $\mathcal{B}$ such that $t p(X) \equiv_{n-k}^{N} t p(Y)$. Note that such an equivalence class $Y$ must exist in $\mathcal{B}$ as otherwise either colored or non-colored disparity would occur in $G_{n-k}\left(\mathcal{A}^{\prime}, \mathcal{B}^{\prime}\right)$ as witnessed by $t p(X)$ and 0 . The case when Spoiler selects an element from $B$ is treated in a similar manner.

On the play $\left(a_{1}, b_{1}\right), \ldots,\left(a_{k}, b_{k}\right),\left(a_{k+1}, b_{k+1}\right)$, the inductive assumption (1) and (2) can be easily checked to hold. To prove that inductive assumption (3) holds, let $\left(i_{k+1}, j_{k+1}\right)$ and $\left(i_{k+1}^{\prime}, j_{k+1}^{\prime}\right)$ be the type of $\left[a_{k+1}\right]$ and $\left[b_{k+1}\right]$ respectively. The strategy ensures one of $\left(i_{k+1}, j_{k+1}\right) \equiv_{n-k}^{C}$ $\left(i_{k+1}^{\prime}, j_{k+1}^{\prime}\right)$ and $\left(i_{k+1}, j_{k+1}\right) \equiv_{n-k}^{N}\left(i_{k+1}^{\prime}, j_{k+1}^{\prime}\right)$ is true, and by Lemma $6,\left(i_{k+1}, j_{k+1}\right) \equiv_{n-k-1}^{C}\left(i_{k+1}^{\prime}, j_{k+1}^{\prime}\right)$ and $\left(i_{k+1}, j_{k+1}\right) \equiv_{n-k-1}^{N}\left(i_{k+1}^{\prime}, j_{k+1}^{\prime}\right)$. It is now routine to show, by using Lemma 6 , that inductive assumption (4) is preserved.

Thus, the strategy is winning for Duplicator by inductive assumptions (1) and (2).

For the next theorem we represent colored equivalence structures $\mathcal{A}$ in three lists. The first one lists equivalence classes of $\mathcal{A}$ in increasing order of their types; the second and the third list the sequences $\left\{q_{(i, j), k}^{\mathcal{A}, C}\right\}_{0 \leq i, j, k \leq n}$ and $\left\{q_{(i, j), k}^{\mathcal{A}, N}\right\}_{0 \leq i, j, k \leq n}$ respectively: 
Theorem 4. Fix $n \in \omega$. There exists an algorithm that runs in constant time and decides whether Duplicator wins the game $G_{n}(\mathcal{A}, \mathcal{B})$ on equivalence structures with one color $\mathcal{A}$ and $\mathcal{B}$. The constant that bounds the running time is $n^{3}$.

Fix $s>1$, let $\mathcal{A}$ be an equivalence structure with $s$ many colors. For each element $x$ of $\mathcal{A}$, define the characteristic of $x$ as defined in the previous sections. There are $2^{s}$ distinct characteristics. Order them in lexicographic order: $c h_{1}, \ldots, c h_{2^{s}}$. Construct the structure $\mathcal{A}^{\prime}=$ $\left(A ; E, Q_{1}, \ldots, Q_{2^{s}}\right)$ such that for all $1 \leq i \leq 2^{s}, Q_{i}=\left\{x \in A \mid c h(x)=c h_{i}\right\}$. Clearly, for distinct characteristics $c h_{i}$ and $c h_{j}$ we have $Q_{i} \cap Q_{j}=\emptyset$. Moreover, $\mathcal{A}$ and $\mathcal{B}$ are isomorphic if and only if $\mathcal{A}^{\prime}$ and $\mathcal{B}^{\prime}$ are isomorphic.

For an equivalence class $X$, we define the type of $X, \operatorname{tp}(X)$, as a sequence $\left(i_{1}, i_{2}, \ldots, i_{2^{s}}\right)$ such that in $X$ the number of element with characteristic $c h_{j}$ is $i_{j}$ for all $1 \leq j \leq 2^{s}$.

Definition 5. Let $\kappa=\left(i_{1}, \ldots, i_{2^{s}}\right)$ and $\lambda=\left(i_{1}^{\prime}, \ldots, i_{2^{s}}^{\prime}\right)$ be two types of equivalence classes. For $1 \leq j \leq 2^{s}$, we say that $\kappa$ is $(j, n)$-equivalent to $\lambda$, denoted by $\kappa \equiv_{n}^{j} \lambda$, if the following holds.

1. If $i_{j}<n$ then $i_{j}^{\prime}=i_{j}$, otherwise $i_{j}^{\prime} \geq n$; and

2. For all $1 \leq l \leq 2^{s}$ where $l \neq j$, if $i_{l}<n-1$ then $i_{l}^{\prime}=i_{l}$, otherwise $i_{l}^{\prime} \geq n-1$.

Let $X$ and $Y$ be equivalence classes of types $\kappa$ and $\lambda$ respectively. If $\kappa \equiv_{n}^{j} \lambda$, then Duplicator wins the $n$-round $\mathrm{EF}$ game played on structures $\left(X ; E \uparrow X, P_{1} \uparrow X, \ldots, P_{s} \uparrow X\right)$ and $(Y ; E \uparrow$ $\left.Y, P_{1} \uparrow Y, \ldots, P_{s} \uparrow Y\right)$, given that Spoiler selects an element $x \in X$ with characteristic $c h_{j}$.

For type $\lambda, 1 \leq j \leq 2^{s}$ and $k \geq 1$, we set $C_{\lambda, k}^{\mathcal{A}, j}$ be the set $\{X \mid X$ is an equivalence class of $\mathcal{A}$ and $\left.t p(X) \equiv_{k}^{j} \lambda\right\}$. Similar to the case of equivalence structures with one color, one introduces notations $q_{\lambda, k}^{A, j}, q_{\lambda, k}^{j}$, and $\mathcal{A}^{j}(\lambda, k)$.

Definition 6. Consider the game $G_{n}(\mathcal{A}, \mathcal{B})$ played on equivalence structures with solors. For $1 \leq j \leq 2^{s}$, we say that a disparity occurs with respect to $c h_{j}$ if there exists a type $\lambda=\left(i_{1}, \ldots, i_{2}\right)$ and $n>k \geq 0$ such that the following holds:

1. $k=q_{\lambda, n-k}^{j}$

2. In one of $\mathcal{A}^{j}(\lambda, n-k)$, there is an equivalence class whose type is $(j, n-k)$-equivalent to $\lambda$, and no such equivalence class exists in the other structure.

The proof of the following are similar to Lemma 7 and Theorem 4 :

Lemma 8. Let $\mathcal{A}$ and $\mathcal{B}$ be equivalence structures with $s$ colors. Duplicator wins the game

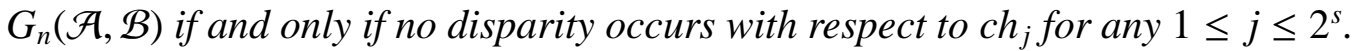

Theorem 5. Fix $n \in \omega$. There exists an algorithm that runs in constant time and decides whether Duplicator wins the game $G_{n}(\mathcal{A}, \mathcal{B})$ on equivalence structures with s colors. The constant that bounds the running time is $n^{2^{s}+1}$. 


\section{Embedded Equivalence Structures}

An embedded equivalence structure of height $h$ is a structure $\mathcal{A}=\left(A ; E_{1}, E_{2}, \ldots, E_{h}\right)$ such that each $E_{i}, 1 \leq i \leq h$, is an equivalence relation, and $E_{i} \subseteq E_{j}$ for $i<j$. In this section we give a full solution for EF played on embedded equivalence structures of height $h$. We start with the case when $h=2$. The case for $h>2$ will be explained later.

Let $\mathcal{A}=\left(A ; E_{1}, E_{2}\right)$ be an embedded equivalence structure of height 2 . We say that an $E_{2^{-}}$ equivalence class $X$ has type $t p(X)=\left(q_{1}, \ldots, q_{t}\right)$ if the largest $E_{1}$-equivalence class contained in $X$ has size $t$ and for all $1 \leq i \leq t, q_{i}$ is the number of $E_{1}$-equivalence classes of size $i$ contained in $X$. Thus, $\sum_{i=1}^{t}\left(q_{i} \times i\right)=|X|$. For two types $\sigma=\left(q_{1}, \ldots, q_{t_{1}}\right)$ and $\tau=\left(q_{1}^{\prime}, \ldots, q_{t_{2}}^{\prime}\right)$, we say $\sigma=\tau$ if $t_{1}=t_{2}$ and $q_{i}=q_{i}^{\prime}$ for all $1 \leq i \leq t_{1}$.

For $X \subseteq A$, we use $\left(X ; E_{1} \uparrow X\right)$ to denote the equivalence structure obtained by restricting $E_{1}$ on $X$. Given two $E_{2}$-equivalence classes $X$ and $Y$ of types $\sigma$ and $\tau$ respectively, we say that $\sigma$ is $n$-equivalent to $\tau$, denoted by $\sigma \equiv_{n} \tau$, if Duplicator wins the $n$-round game played on structures $\left(X ; E_{1} \uparrow X\right)$ and $\left(Y ; E_{1} \uparrow Y\right)$. Note that if $\sigma \equiv_{n} \tau$, then $\sigma \equiv_{i} \tau$ for all $i \leq n$.

We need the following notations:

- For type $\sigma$ and $i \geq 1$, set $C_{\sigma, i}^{\mathcal{A}}$ be the set $\left\{X \mid X\right.$ is an $E_{2}$-equivalence class of $\mathcal{A}$ and $\left.\operatorname{tp}(X) \equiv_{i} \sigma\right\}$.

- Set $q_{\sigma, i}^{\mathcal{A}}=\left|C_{\sigma, i}^{\mathcal{A}}\right|$.

- For embedded equivalence structure $\mathcal{A}$ and $\mathcal{B}$, set $q^{\sigma, i}=\min \left\{q_{\sigma, i}^{\mathcal{A}}, q_{\sigma, i}^{\mathcal{B}}\right\}$

- Set $\mathcal{A}(\sigma, i)$ be the embedded equivalence structure of height 2 obtained from $\mathcal{A}$ by removing $q^{\sigma, i}$ equivalence classes whose types are $i$-equivalent to $\sigma$.

Observe in round $k$ of the game $G_{n}(\mathcal{A}, \mathcal{B})$, if Spoiler selects an element from an $E_{2^{-}}$ equivalence class $X$ in $\mathcal{A}$, and Duplicator responds by selecting another element from an $E_{2}$-equivalence class $Y$ in $\mathcal{B}$ such that $t p(Y) \equiv_{n-k} t p(X)$, there is no point for Spoiler to keep playing inside $X$ because this will guarantee a win for Duplicator. Intuitively, $\mathcal{A}(\sigma, n-k)$ contains all the $E_{2}$-equivalence classes for Spoiler to choose elements from after $q^{\sigma, n-k}$ many $E_{2}$-equivalence classes whose types are $(n-k)$-equivalent to $\sigma$ have been chosen.

Definition 7. Consider the game $G_{n}(\mathcal{A}, \mathcal{B})$ played on embedded equivalence structures of height 2. We say that a disparity occurs if there exists a type $\sigma$ and $n>k \geq 0$ such that the following holds.

1. $k=q^{\sigma, n-k}$.

2. In one of $\mathcal{A}(\sigma, n-k)$ and $\mathcal{B}(\sigma, n-k)$, there is an $E_{2}$-equivalence class whose type is $(n-k)$-equivalent to $\sigma$, and no such $E_{2}$-equivalence class exists in the other structure.

Lemma 9. Suppose $\mathcal{A}$ and $\mathcal{B}$ are two embedded equivalence structures of height 2. Duplicator wins the game $G_{n}(\mathcal{A}, \mathcal{B})$ if and only if no disparity occurs.

Proof. Suppose disparity occurs in $G_{n}(\mathcal{A}, \mathcal{B})$ witnessed by $\sigma$ and $k$, in $\mathcal{A}(\sigma, n-k)$ there is an $E_{2}$-equivalence class whose type is $(n-k)$-equivalent to $\sigma$, and no such $E_{2}$-equivalence class exists in $\mathcal{B}(\sigma, n-k)$. Using these, it is not hard to prove that Spoiler wins the game. 
Suppose that no disparity occurs in the game $G_{n}(\mathcal{A}, \mathcal{B})$, we describe a strategy for Duplicator. Let us assume that the players have produced a $k$-round play $\left(a_{1}, b_{1}\right),\left(a_{2}, b_{2}\right), \ldots$, $\left(a_{k}, b_{k}\right)$. Let $\sigma_{i}$ and $\tau_{i}$ be the types of $a_{i}$ and $b_{i}$, respectively with $1 \leq i \leq k$. Our inductive assumptions on this $k$-round play are the following:

1. The map $a_{i} \rightarrow b_{i}$ is partial isomorphism.

2. For all $1 \leq i \leq k, \sigma_{i} \equiv_{n-i} \tau_{i}$.

3. Let $\mathcal{A}^{\prime}$ and $\mathcal{B}^{\prime}$ be the equivalence structures obtained by removing the $E_{2}$-equivalence classes $\left[a_{1}\right]_{E_{2}}, \ldots,\left[a_{k}\right]_{E_{2}}$ from $\mathcal{A}$ and the equivalence classes $\left[b_{1}\right]_{E_{2}}, \ldots,\left[b_{k}\right]_{E_{2}}$ from $\mathcal{B}$, respectively. We assume in game $G_{n-k}\left(\mathcal{A}^{\prime}, \mathcal{B}^{\prime}\right)$ no disparity occurs.

Assume that Spoiler selects an element $a_{k+1} \in A$. The case when Spoiler selects an element from $B$ is treated as below. Duplicator responds to this move by choosing $b_{k+1}$ as follows. If $a_{k+1}=a_{i}$ then $b_{k+1}=b_{i}$. Otherwise, if $E_{1}\left(a_{i}, a_{k+1}\right)$ is true in $\mathcal{A}$, then Duplicator chooses a new $b_{k+1}$ such that $E_{1}\left(b_{i}, b_{k+1}\right)$. If $E_{2}\left(a_{i}, a_{k+1}\right)$ is true in $\mathcal{A}$ and there is no $j$ such that $E_{1}\left(a_{j}, a_{k+1}\right)$, then Duplicator chooses a new $b_{k+1}$ such that $E_{2}\left(b_{i}, b_{k+1}\right)$ and there is no $j$ such that $E_{1}\left(b_{j}, b_{k+1}\right)$. By (2) of the inductive assumption Duplicator can always select such an element $b_{k+1}$ by following its winning strategies.

Assume $a_{k+1}$ is not equivalent to any of the elements $a_{1}, \ldots, a_{k}$. Let $X$ be the $E_{2}$-equivalence class in $\mathcal{A}$ that contains $a_{k+1}$. Duplicator selects $b_{k+1}$ from an $E_{2}$-equivalence class $Y$ in $\mathcal{B}$ such that $t p(X) \equiv_{n-k} t p(Y)$. Duplicator is able to select such an element as otherwise disparity would occur as witnessed by the type of $X$ and 0 .

The inductive assumption (1) and (2) can be easily checked to hold on the play $\left(a_{1}, b_{1}\right), \ldots$, $\left(a_{k}, b_{k}\right),\left(a_{k+1}, b_{k+1}\right)$. To show that the assumption (3) is preserved, consider the structures $\mathcal{A}^{\prime \prime}$ and $\mathcal{B}^{\prime \prime}$ obtained by removing $\left[a_{1}\right]_{E_{2}}, \ldots,\left[a_{k}\right]_{E_{2}},\left[a_{k+1}\right]_{E_{2}}$ and $\left[b_{1}\right]_{E_{2}}, \ldots,\left[b_{k}\right]_{E_{2}},\left[b_{k+1}\right]_{E_{2}}$ from $\mathcal{A}$ and $\mathcal{B}$, respectively. Suppose disparity occurs in $G_{n-k-1}\left(\mathcal{A}^{\prime \prime}, \mathcal{B}^{\prime \prime}\right)$ as witnessed by some type $\tau$ and $t<n-k-1$. There are two cases. If $t p\left(\left[a_{k+1}\right]\right) \equiv_{n-k-t-1} \tau$, then $t p\left(\left[b_{k+1}\right]\right) \equiv_{n-k-t-1} \tau$, and disparity must occur in $G_{n-k}\left(\mathcal{A}^{\prime}, \mathcal{B}^{\prime}\right)$ as witnessed by $\tau$ and $t+1$. If $t p\left(\left[a_{k+1}\right]\right) \not_{n-k-t-1} \tau$, then $t p\left(\left[b_{k+1}\right]\right) \not_{n-k-t-1} \tau$, and disparity must occur in $G_{n-k}\left(\mathcal{A}^{\prime}, \mathcal{B}^{\prime}\right)$ as witnessed by $\tau$ and $t$, contradicting our assumption. Hence the strategy is a winning strategy.

Theorem 6. Fix $n \in \omega$. There exists an algorithm that runs in constant time and decides whether Duplicator wins the game $G_{n}(\mathcal{A}, \mathcal{B})$ on embedded equivalence structures of height 2. The constant that bounds the running time is $(n+1)^{n}$.

Proof. We represent structure $\mathcal{A}=\left(A ; E_{1}, E_{2}\right)$ by a tree and a list. The tree has height 3 . The leaves of the tree are all elements in $A$. Two leaves $x, y$ have the same parent if $E_{1}(x, y)$, and $x, y$ have the same ancestor at level 1 if $E_{2}(x, y)$. Intuitively, we can view the root of tree as $A$, the internal nodes at level 1 represent all $E_{2}$-equivalence classes on $A$, and the children of each $E_{2}$-equivalence class $X$ at level 2 are all $E_{1}$-equivalence classes contained in $X$. We further require that representations of $E_{2}$ and $E_{1}$-equivalence classes are put in left-to-right order according to their cardinalities.

The list is $q_{\sigma_{1}, 1}^{\mathcal{P}}, \ldots, q_{\sigma_{t}, 1}^{\mathcal{A}}, \ldots, q_{\sigma_{1}, n}^{\mathcal{A}}, \ldots, q_{\sigma_{t}, n}^{\mathcal{A}}$ where each $\sigma_{i}$ is a type of $E_{2}$-equivalence class, and $q_{\sigma_{i}, j}^{\mathcal{A}}$ is as defined above. Each $q_{\sigma_{i}, j}^{\mathcal{A}}$ has a value between 0 and $n$ and if it is greater 
than $n$, we set it to $n$. The algorithm checks whether disparity occurs in $G_{n}(\mathcal{A}, \mathcal{B})$ by examining the list. There can be at most $(n+1)^{n}$ pairwise non- $n$-equivalent types. Therefore, checking disparity requires a time bounded by $(n+1)^{n+1}$.

For the case when $\mathcal{A}$ and $\mathcal{B}$ are two embedded equivalence structures of height $h$, where $h>2$, we give a similar definition of the type of an $E_{h}$-equivalence class. We can then describe the winning conditions for Spoiler and Duplicator in a similar way.

Let $\mathcal{A}$ be an embedded equivalence structure of height $h$ where $h>2$. For an $E_{h^{-}}$ equivalence class $X$, we recursively define $t p(X)$, the type of $X$. Set $t p(X)$ be $\left(q_{\sigma_{1}}, \ldots, q_{\sigma_{t}}\right)$ that satisfies the following property.

1. Each $\sigma_{i}$ is the type of an $E_{h-1}$-equivalence class.

2. $\sigma_{t}$ is the maximum type in lexicographic order among all types of $E_{h-1}$-equivalence classes contained in $X$.

3. The list $\sigma_{1}, \ldots, \sigma_{t}$ contains all possible types of $E_{h-1}$-equivalence classes less or equal to $\sigma_{t}$ ordered lexicographically.

4. For all $1 \leq i \leq t, q_{\sigma_{i}}$ is the number of all $E_{h-1}$-equivalence classes contained in $X$ whose type are $\sigma_{i}$.

We note that these types allow us to solve the isomorphism problem for embedded equivalence structures of height $h$ in linear time on the size of the structures.

Let $\kappa=\left(q_{\sigma_{1}}, \ldots, q_{\sigma_{s}}\right)$ and $\lambda=\left(q_{\sigma_{1}}^{\prime}, \ldots, q_{\sigma_{t}}^{\prime}\right)$ be types of two $E_{h}$-equivalence classes $X$ and $Y$, respectively. We say $\kappa=\lambda$ if $s=t$ and $q_{\sigma_{i}}=q_{\sigma_{i}}^{\prime}$ for all $1 \leq i \leq s$. We say $\kappa \equiv_{n} \lambda$ if the structures $\left(X ; E_{1} \uparrow X, \ldots, E_{h-1} \uparrow X\right)$ and $\left(Y ; E_{1} \uparrow Y, \ldots, E_{h-1} \uparrow Y\right)$ are $n$-equivalent.

Similarly to the case of embedded equivalence structures of height 2 , we re-introduce the notions $C_{\sigma, i}^{\mathcal{A}}, q_{\sigma, i}^{\mathcal{A}}, q^{\sigma, i}, \mathcal{A}(\sigma, i)$ and disparity in the game $G_{n}(\mathcal{A}, \mathcal{B})$.

Lemma 10. Suppose $\mathcal{A}$ and $\mathcal{B}$ are two embedded equivalence structures of height $h$ where $h \geq 2$. Duplicator wins the game $G_{n}(\mathcal{A}, \mathcal{B})$ if and only if no disparity occurs.

The number of pairwise non- $n$-equivalent types of $E_{h}$-equivalence classes is at most $(n+$ 1) $\cdots^{(n+1)^{n}}$ where the tower of $(n+1)$ has height $h$. Thus, by the lemma above, we have:

Theorem 7. Fix $n \in \omega$. There is an algorithm that runs in constant time and decides if Duplicator wins the game $G_{n}(\mathcal{A}, \mathcal{B})$ on embedded equivalence structures $\mathcal{A}$ and $\mathcal{B}$ of height $h$. The constant that bounds the running time is $(n+1)^{\cdots^{(n+1)}}$ where the tower has height $h$.

\section{Trees}

In this section we are interested in trees; these are finite structures of the type $\mathcal{T}=(T, \leq)$, where the relation $\leq$ is a partial order on $T$ such that $\mathcal{T}$ has the greatest element (the root), and the set $\{y \mid x \leq y\}$ for any given $x \in T$ is a linearly ordered set under $\leq$. We call an element a leaf of the tree $\mathcal{T}$ if it is a minimal element; otherwise we call it an internal node. A path in $\mathcal{T}$ is a maximal linearly order subset of $T$. The length of a given path is the number of elements in the path. The height of $\mathcal{T}$ is the length of the largest path in $\mathcal{T}$. 
We say that the level of a node $x \in T$ is $j$ if the distance from $x$ to the root is $j$. We fix number $h \geq 2$, and restrict ourselves to the class $\mathcal{K}_{h}$ of all trees of height at most $h$. Deciding Ehrenfeucht-Fraïssé games on trees from $\mathcal{K}_{h}$ can be done directly by using the techniques from the previous section. Instead, we reduce the problem of deciding Ehrenfeucht-Fraïssé games on trees in $K_{h}$ to one for embedded equivalence structures of height $h+1$.

We transform trees from the class $\mathcal{K}_{h}$ into the class of embedded equivalence structures of height $h$ in the following manner. Let $\mathcal{T}$ be a tree in $\mathcal{K}_{h}$. We now define an embedded equivalence structure $\mathcal{A}(\mathcal{T})$ as follows. The domain $D$ of $\mathcal{A}(T)$ is now $T \cup\left\{a_{x} \mid x\right.$ is a leaf of $\mathcal{T}\}$. We define the equivalence relation $E_{i}, 1 \leq i \leq h$, on the domain as follows. The relation $E_{1}$ is the minimal equivalence relation that contains $\left\{\left(x, a_{x}\right) \mid x\right.$ is a leaf of $\left.\mathcal{T}\right\}$. Let $x_{1}, \ldots, x_{s}$ be all elements of $\mathcal{T}$ at level $h-i+1$, where $1 \leq i<h$. Let $\mathcal{T}_{1}, \ldots, \mathcal{T}_{s}$ be the subtrees of $\mathcal{T}$ whose roots are $x_{1}, \ldots, x_{s}$, respectively. Set $E_{i}$ be the minimal equivalence relation that contains $E_{i-1} \cup T_{1}^{2} \cup \ldots \cup T_{s}^{2}$. It is clear that $E_{i} \subseteq E_{i+1}$ for all $1 \leq i \leq h$. Thus we have the embedded equivalence structure $\mathcal{A}(T)=\left(D ; E_{1}, \ldots, E_{h}\right)$.

Lemma 11. For trees $\mathcal{T}_{1}$ and $\mathcal{T}_{2}, \mathcal{T}_{1} \cong \mathcal{T}_{2}$ if and only if $\mathcal{A}\left(\mathcal{T}_{1}\right) \cong \mathcal{A}\left(\mathcal{T}_{2}\right)$. In particular, Duplicator wins $G_{n}\left(\mathcal{T}_{1}, \mathcal{T}_{2}\right)$ if and only if Duplicator wins $G_{n}\left(\mathcal{A}\left(\mathcal{T}_{1}\right), \mathcal{A}\left(T_{2}\right)\right)$.

Proof. Suppose $\mathcal{T}$ is a tree in the class $\mathcal{K}_{h}$. Take an element $x \in T$. By construction of $\mathcal{A}(\mathcal{T})$, the following statements are true.

- $x$ is a leaf in $\mathcal{T}$ if and only if $\left|\left\{y \mid E_{1}(x, y)\right\}\right|=2$ in $\mathcal{A}(\mathcal{T})$.

$-x$ is the root of $\mathcal{T}$ if and only if $\left|\left\{y \mid E_{h}(x, y)\right\}\right|=1$ in $\mathcal{A}(\mathcal{T})$.

We define the level of $x$ in $\mathcal{A}(\mathcal{T})$ as follows. If $x$ is the root of $\mathcal{T}$, the level of $x$ is 0 . Otherwise, if $x$ is an internal node, the level of $x$ in $\mathcal{A}(\mathcal{T})$ is the largest $l$ such that $\mid\{y \mid$ $\left.E_{h-l+1}(x, y)\right\} \mid>1$. If $x$ is a leaf, we define the level of $x$ in $\mathcal{A}(\mathcal{T})$ to be the largest $l+1$ such that there is an internal node $y$ such that $E_{h-l+1}(x, y)$.

By definition, for all $x \in T$, the level of $x$ in $\mathcal{T}$ is the level of $x$ in $\mathcal{A}(\mathcal{T})$. For $x, y \in T$, $x \leq y$ in $\mathcal{T}$ if and only in $\mathcal{A}(\mathcal{T}) x$ has level $s$ and $y$ has level $t$ such that $s \geq t$ and $E_{h-t+1}(x, y)$. Thus, for two trees from $\mathcal{K}_{h}, \mathcal{T}_{1}$ and $\mathcal{T}_{2}$, and a mapping $f: T_{1} \rightarrow T_{2}, f$ is an isomorphism between $\mathcal{T}_{1}$ and $\mathcal{T}_{2}$ if and only if $f$ is an isomorphism between $\mathcal{A}\left(\mathcal{T}_{1}\right)$ and $\mathcal{A}\left(\mathcal{T}_{2}\right)$.

To prove the second part of the lemma, one direction is clear. For the other direction, assume that there is a winning strategy for Duplicator on the game $G_{n}\left(\mathcal{T}_{1}, \mathcal{T}_{2}\right)$. We describe a strategy for Duplicator on the game $G_{n}\left(\mathcal{A}\left(\mathcal{T}_{1}\right), \mathcal{A}\left(\mathcal{T}_{2}\right)\right)$ where $\mathcal{A}\left(\mathcal{T}_{1}\right)=\left(D_{1} ; E_{1}, \ldots, E_{h}\right)$ and $\mathcal{A}\left(\mathcal{T}_{2}\right)=\left(D_{2} ; E_{1}, \ldots, E_{h}\right)$. Let us assume that the players have produced a $k$-round play $\left(x_{1}, y_{1}\right),\left(x_{2}, y_{2}\right), \ldots,\left(x_{k}, y_{k}\right)$. Assume on this $k$-round play the map $x_{i} \rightarrow y_{i}$ is a partial isomorphism between $\mathcal{A}\left(\mathcal{T}_{1}\right)$ and $\mathcal{A}\left(\mathcal{T}_{2}\right)$.

Assume that Spoiler selects an element $x_{k+1} \in D_{1}$. Duplicator responds to this move by choosing $x_{k+1}$ as follows. If $x_{k+1}=x_{i}$ then $y_{k+1}=y_{i}$. Otherwise, if $x_{k+1} \in T_{1}$, then Duplicator selects an element $y_{k+1} \in T_{2}$ according to its winning strategy on $G_{n}\left(\mathcal{T}_{1}, \mathcal{T}_{2}\right)$. If $x_{k+1}=a_{x}$ for some leaf $x \in \mathcal{T}_{1}$. Then Duplicator responds by selecting $y_{k+1}=a_{y}$ where $y$ is the leaf in $T_{2}$ that corresponds to $x$ in Duplicator's winning strategy in $G_{n}\left(\mathcal{T}_{1}, \mathcal{T}_{2}\right)$. It is clear that $x_{i} \rightarrow y_{i}$ 
where $1 \leq i \leq k+1$ is also a partial isomorphism between $\mathcal{A}\left(\mathcal{T}_{1}\right)$ and $\mathcal{A}\left(\mathcal{T}_{2}\right)$. Therefore the strategy described is a winning strategy for Duplicator on game $G_{n}\left(\mathcal{A}\left(\mathcal{T}_{1}\right), \mathcal{A}\left(\mathcal{T}_{2}\right)\right)$.

Using the lemma above, one can now prove this:

Theorem 8. Fix $n \in \omega$. There is an algorithm that runs in constant time and decides if Duplicator wins the game $G_{n}\left(\mathcal{T}_{1}, \mathcal{T}_{2}\right)$, where $\mathcal{T}_{1}, \mathcal{T}_{2} \in \mathcal{K}_{h}$. The constant that bounds the running time is $(n+1)^{\ldots^{(n+1))^{(n+1)}}}$ where the tower has height $h$.

\section{Boolean Algebras with Distinguished Ideals}

A Boolean algebra (BA) with distinguished ideals is a structure $\mathcal{A}=\left(A ; \leq, 0,1, I_{1}, \ldots, I_{s}\right)$, where $(A ; \leq, 0,1)$ forms a BA and each $I_{j}$ is an ideal of the algebra $(A ; \leq, 0,1)$. The set of atoms of $\mathcal{A}$, denoted $A t(\mathcal{A})$, is the set $\{a \mid \forall y(0 \leq y \leq a \rightarrow y=0 \vee y=a)\}$. Since we restrict ourselves to finite structures, the BA $(A ; \leq, 0,1)$ can be identified with the structure $\left(2^{X_{A}} ; \subseteq, \emptyset, X_{A}\right)$, where $X_{A}=A t(\mathcal{A})$ and $2^{X_{A}}$ is the collection of all subsets of $X_{A}$. Moreover, for each ideal $I_{j}$ there exists a set $A_{j} \subset A t(\mathcal{A})$ such that $I_{j}=2^{A_{j}}$. Hence the original structure $\mathcal{A}$ can be identified with the structure: $\left(2^{X_{A}} ; \subseteq, \emptyset, X_{A}, 2^{A_{1}}, \ldots, 2^{A_{s}}\right)$. For each element $x \in \operatorname{At}(\mathcal{A})$, define the characteristic of $x, \operatorname{ch}(x)$, as a binary sequence $\left(t_{1}, t_{2}, \ldots, t_{s}\right)$ such that for each $1 \leq i \leq s, t_{i} \in\{0,1\}, t_{i}=1$ if $x \in A_{i}$ and $t_{i}=0$ otherwise. For each characteristic $\epsilon \in\{0,1\}^{s}$ consider the set $\left.A_{\epsilon}=\{x \in \operatorname{At}(\mathcal{A}) \mid \operatorname{ch}(x)=\epsilon)\right\}$. This defines the ideal $I_{\epsilon}$ in the Boolean algebra $\left(2^{X_{A}} ; \subseteq, \emptyset, X_{A}\right)$. Moreover, we can also identify this ideal with the Boolean algebra $\left(2^{A_{\epsilon}} ; \subseteq, \emptyset, A_{\epsilon}\right)$. There are $2^{s}$ pairwise distinct characteristics. Let $\epsilon_{1}, \ldots, \epsilon_{2^{s}}$ be the list of all characters. We denote by $\mathcal{A}^{\prime}$ the following structure: $\left(2^{X} ; \subseteq, \emptyset, X, 2^{A_{\epsilon_{1}}}, \ldots, 2^{A_{\epsilon_{2} s}}\right)$.

Lemma 12. Let $\mathcal{A}=\left(2^{X_{A}} ; \subseteq, \emptyset, X_{A}, 2^{A_{1}}, \ldots, 2^{A_{s}}\right)$ be a Boolean algebra with distinguished ideals

1. For any two distinct characteristics $\epsilon$ and $\delta$ we have $I_{\epsilon} \cap I_{\delta}=\{\emptyset\}$.

2. For any element $a \in 2^{X}$ there are elements $a_{\epsilon} \in I_{\epsilon}$ such that $a=\cup_{\epsilon} a_{\epsilon}$.

3. The Boolean algebra $\left(2^{X_{A}} ; \subseteq, \emptyset, X_{A}\right)$ is isomorphic to the Cartesian product of the Boolean algebras $I_{\epsilon}$.

4. $\mathcal{A}$ and $\mathcal{B}$ are isomorphic if and only if $\mathcal{A}^{\prime}$ and $\mathcal{B}^{\prime}$ are isomorphic.

The next lemma connects the structure $\mathcal{A}^{\prime}$ and $\mathcal{A}$ in terms of characterizing the winner of the game $G_{n}(\mathcal{A}, \mathcal{B})$.

Lemma 13. Duplicator wins the game $G_{n+1}(\mathcal{A}, \mathcal{B})$ if and only if each of the following two conditions are true:

1. For each characteristic $\epsilon,\left|A_{\epsilon}\right| \geq 2^{n}$ if and only if $\left|B_{\epsilon}\right| \geq 2^{n}$.

2. For each characteristic $\epsilon$, if $\left|A_{\epsilon}\right|<2^{n}$ then $\left|A_{\epsilon}\right|=\left|B_{\epsilon}\right|$.

Proof. Assume that for some $\epsilon$, we have $\left|A_{\epsilon}\right| \neq\left|B_{\epsilon}\right|$ and $\left|B_{\epsilon}\right|<2^{n}$. Let us assume that $\left|A_{\epsilon}\right| \geq 2^{n}$. The case when $\left|A_{\epsilon}\right|<2^{n}$ is treated in a similar manner. We describe a winning strategy for Spoiler. Spoiler starts by taking elements $a_{1}, a_{2}, \ldots$ in $A_{\epsilon}$. For each $i \leq n$ the 
element $a_{i}$ is such that $\left|\operatorname{At}\left(a_{i}\right)\right| \geq 2^{n-i}$, where $\operatorname{At}(a)$ denotes the set of atoms below $a$. The elements $a_{1}, a_{2}, \ldots$ are such that for each $i$, either $a_{i} \subset a_{i-1}$ or $a_{i} \cap a_{i-1}=\emptyset$. Consider the $k$ round play $\left(a_{1}, b_{1}\right), \ldots,\left(a_{k}, b_{k}\right)$ where $k<n$. Let $e<k$ be the last round for which $a_{k} \subset a_{e}$. If no such $e$ exists, let $a_{e}=2^{A_{\epsilon}}$ and $b_{e}=2^{B_{\epsilon}}$. We have the following inductive assumptions.

- $\left|\operatorname{At}\left(a_{k}\right)\right| \geq 2^{n-k}$ and $\left|\operatorname{At}\left(a_{e} \backslash\left(a_{e+1} \cup \ldots \cup a_{k}\right)\right)\right| \geq 2^{n-k}$.

- Either $\left|A t\left(b_{k}\right)\right|<2^{n-k}$ or $\left|A t\left(b_{e} \backslash\left(b_{e+1} \cup \ldots \cup b_{k}\right)\right)\right|<2^{n-k}$.

There are two cases.

Case 1. Assume that $\left|A t\left(b_{k}\right)\right|<2^{n-k}$ and $\left|A t\left(a_{k}\right)\right| \geq 2^{n-k}$. In this case Spoiler selects $a_{k+1}$ such that $a_{k+1} \subset a_{k}, a_{k+1} \neq \emptyset,\left|A t\left(a_{k+1}\right)\right| \geq 2^{n-k-1}$ and $\left|A t\left(a_{k} \mid a_{k+1}\right)\right| \geq 2^{n-k-1}$. Note that Duplicator must choose $b_{k+1}$ strictly below $b_{k}$. Then either $\left|A t\left(b_{k+1}\right)\right|<2^{n-k-1}$ or $\mid A t\left(b_{k} \mid\right.$ $\left.b_{k+1}\right) \mid<2^{n-k-1}$

Case 2. Assume that $\left|A t\left(b_{k}\right)\right| \geq 2^{n-k}$ and $\left|A t\left(a_{k}\right)\right| \geq 2^{n-k}$. In this case, Spoiler selects $a_{k+1}$ such that $a_{k+1} \subset a_{e}, a_{k+1} \neq \emptyset, a_{k+1} \cap\left(a_{e+1} \cup \ldots \cup a_{k}\right)=\emptyset,\left|\operatorname{At}\left(a_{k+1}\right)\right| \geq 2^{n-k-1}$, and $\mid \operatorname{At}\left(a_{e} \backslash\left(a_{e+1} \cup\right.\right.$ $\left.\left.\ldots \cup a_{k+1}\right)\right) \mid \geq 2^{n-k-1}$. Note that by definition of $e,\left|A t\left(b_{e}\right)\right|<2^{n-k}$ and for each $e+1 \leq i \leq k-1$, $\left|A t\left(b_{i}\right)\right| \geq 2^{n-i}$ as otherwise $b_{k}$ would be below $b_{i}$. Hence $\left|A t\left(b_{k} \backslash\left(b_{e+1} \cup \ldots \cup b_{k}\right)\right)\right|<2^{n-k}$. Duplicator must choose $b_{k+1}$ strictly below $b_{e}$ and disjoint with $b_{e+1}, \ldots, b_{k}$. Therefore either $\left|A t\left(b_{k+1}\right)\right|<2^{n-k-1}$ or $\left|A t\left(b_{e}\right) \backslash A t\left(b_{e+1} \cup \ldots \cup b_{k+1}\right)\right|<2^{n-k-1}$.

After $n$ rounds, by the inductive assumption, it is either $\left|A t\left(b_{n}\right)\right|=0$ or $\mid A t\left(b_{e} \backslash\left(b_{e+1} \cup \ldots \cup\right.\right.$ $\left.\left.b_{n}\right)\right) \mid=0$. If the former, then Spoiler wins by selecting $a_{n+1} \subset A t\left(a_{n}\right)$;otherwise, Spoiler wins by selecting $a_{n+1} \subset a_{e} \backslash\left(a_{e+1} \cup \ldots \cup a_{n}\right)$.

Now we prove that the conditions stated in the lemma suffice Duplicator to win the $(n+$ 1)-round game $G_{n+1}(\mathcal{A}, \mathcal{B})$. Let us assume that the players have produced a $k$-round play $\left(a_{1}, b_{1}\right),\left(a_{2}, b_{2}\right), \ldots,\left(a_{k}, b_{k}\right)$. Our inductive assumptions on this $k$-round play are the following:

1. The map $a_{i} \rightarrow b_{i}$ is a partial isomorphism.

2. For each $a_{i}, 1 \leq i \leq k$, let $a_{i}=\cup_{\epsilon} a_{\epsilon}$ be as stipulated in Lemma 12(2). For each $a_{\epsilon}$, let $e$ be the last round such that $a_{\epsilon} \subseteq a_{e}$, if there is no such round, then assume $a_{e}=\operatorname{At}\left(I_{\epsilon}\right)$. Let $d$ be the last round such that $a_{d} \subseteq a_{\epsilon}$, if there is no such round, then assume $a_{d}=\emptyset$. Let $b_{i}=\cup_{\epsilon} b_{\epsilon}$. The conditions for $b_{\epsilon}$ are the following:

- $\left|\operatorname{At}\left(a_{\epsilon} \backslash a_{d}\right)\right| \geq 2^{n-i}$ if and only if $\left|\operatorname{At}\left(b_{\epsilon} \backslash a_{d}\right)\right| \geq 2^{n-i} ;\left|\operatorname{At}\left(a_{e} \backslash a_{\epsilon}\right)\right| \geq 2^{n-i}$ if and only if $\left|A t\left(b_{e} \backslash b_{\epsilon}\right)\right| \geq 2^{n-i}$.

- If $\left|\operatorname{At}\left(a_{\epsilon} \backslash a_{d}\right)\right|<2^{n-i}$ then $\left|\operatorname{At}\left(b_{\epsilon} \backslash a_{d}\right)\right|=\left|\operatorname{At}\left(a_{\epsilon} \backslash a_{d}\right)\right|$; If $\left|\operatorname{At}\left(a_{e} \backslash a_{\epsilon}\right)\right|<2^{n-i}$ then $\left|\operatorname{At}\left(b_{e} \backslash b_{\epsilon}\right)\right|=\left|\operatorname{At}\left(a_{e} \backslash a_{\epsilon}\right)\right|$.

Assume that Spoiler selects an element $a_{k+1} \in A$. Duplicator responds to this move by choosing $b_{k+1}$ as follows. If $a_{k+1}=a_{i}$ then $b_{k+1}=b_{i}$. Otherwise, suppose $a_{k+1}=\cup a_{\epsilon}$ as stipulated in Lemma 12(2). For each $a_{\epsilon}$, let $d, e$ be as described in the inductive assumptions. We select each $b_{\epsilon}$ by the following rules.

- If $\left|\operatorname{At}\left(a_{\epsilon} \backslash a_{d}\right)\right| \geq 2^{n-k-1}$ then select $b_{\epsilon}$ such that $\left|\operatorname{At}\left(b_{\epsilon} \backslash a_{d}\right)\right| \geq 2^{n-k-1}$; If $\left|\operatorname{At}\left(a_{e} \backslash a_{\epsilon}\right)\right| \geq 2^{n-k-1}$ then $\left|A t\left(b_{e} \backslash b_{\epsilon}\right)\right| \geq 2^{n-k-1}$.

- If $\left|\operatorname{At}\left(a_{\epsilon} \backslash a_{d}\right)\right|<2^{n-k-1}$ then select $b_{\epsilon}$ such that $\left|\operatorname{At}\left(b_{\epsilon} \backslash a_{d}\right)\right|=\left|\operatorname{At}\left(a_{\epsilon} \backslash a_{d}\right)\right| ;$ If $\left|\operatorname{At}\left(a_{e} \backslash a_{\epsilon}\right)\right|<$ $2^{n-k-1}$ then $\left|A t\left(b_{e} \backslash b_{\epsilon}\right)\right|=\left|A t\left(a_{e} \backslash a_{\epsilon}\right)\right|$. 
Finally, Duplicator selects $b_{k+1} \in B$ such that $b_{k+1}=\cup_{\epsilon} b_{\epsilon}$.

Note the inductive assumptions guarantee that Duplicator is able to make such a move. It is clear that the inductive assumptions also hold on the $(k+1)$-round play $\left(a_{1}, b_{1}\right), \ldots,\left(a_{k+1}, b_{k+1}\right)$. Hence, the strategy described must be a winning strategy due to the fact that Duplicator preserves the inductive assumption (1) at each round. The lemma is proved.

For the next result, we represent the Boolean algebras by listing their atoms in $2^{s}$ lists, where the $i^{\text {th }}$ list lists all atoms with characteristic $\epsilon_{i}$ :

Theorem 9. Fix $n \in \omega$. There exists an algorithm that runs in constant time and decides whether Duplicator wins the game $G_{n+1}(\mathcal{A}, \mathcal{B})$ on $B A s \mathcal{A}$ and $\mathcal{B}$ with s distinguished ideals. The constant that bounds the running time is $2^{s} \cdot 2^{n}$.

\section{References}

1. S. Arora, R. Fagin, On winning strategies in EF games. Theoretical Computer Science 174, 97-121, 1997.

2. A. Dawar, Infinitary logic and inductively definability over finite structures. Information and Computation, volume 119, 2:160-175, 1995.

3. A. Ehrenfeucht, An appliction of games to the completeness problem for formalized theories. Fundamenta Mathematicae,49:129-141,1961.

4. R. Fraïssé, Sur quelques classfications des systèm de relations. Université d Alger, Publication Scientifiques, Sér. A,1:35-182, 1954.

5. M. Grohe. Equivalence in finite-variable logics is complete for polynomial time. Proceeding FOCS 96.

6. W. Hodeges, Model theory, Combridge University Press, 1993.

7. N. Immerman, D. Kozen, Definability with bounded number of bounded variables. Logic in Computer Science, 236244, 1987.

8. C. Karp, Finite quantifier equivalence, In J.W. Addison, L. Henkin, and A. Tarski, editors, The Theory of Models, 407-412. North Holland, 1965.

9. P. Kolaitis, J. Panttaja, On the complexity of existential pebble games. In M. Baaaz and J.A. Makowsky (Eds): CSL 2003, LNCS2803, pp. 314-329, 2003.

10. C. Lautemann, N. Schweikardt, An EF approach to collapse results for first-order queries over embedded databases. In A. Ferreira and H. Reichel, editors, STACS'01:18th Annual Symposium on Theoretical Aspects of Computer Science, volume 2010 of Lecture Notes in Computer Science, 455-466, Dresden, Germany, Springer, 2001.

11. L. Libkin, Elements of finite model theory. Springer-Verlag, 2004.

12. J. Marcinkowski, Directed Reachability: From Ajtai-Fagin to Ehrenfeucht-Fraïssé games. Proceedings of CSL99, Springer LNCS, 1999.

13. A. Mekler, S. Shelah, J. Väänänen, The EF-game of length $\omega_{1}$. Transactions of the American mathematical Society, Volume 339, Number 2, 1993.

14. E. Pezzoli, Computational complexity of EF games on finite structures. In Computer Science Logic, 12th International Workshop, CSL'98, 159-170, 1999.

15. D. Scott, Logic with denumerable long formulas and finite strings of quantifiers. In J.W. Addison, L. Henkin, and A. Tarski, editors, The Theory of Models, 329-341. North Holland, 1965.

16. N. Schweikardt, An EF game appraoch to collapse results in database theory. To appear in Information and Computation, 2006. 